hep-ph/0607002

CERN-PH-TH/2006-110

UMN-TH-2509/06

FTPI-MINN-06/23

\title{
What if supersymmetry breaking appears below the GUT scale?
}

\author{
John Ellis ${ }^{1}$, Keith A. Olive ${ }^{2}$ and Pearl Sandick ${ }^{2}$ \\ ${ }^{1}$ TH Division, CERN, Geneva, Switzerland \\ ${ }^{2}$ William I. Fine Theoretical Physics Institute, \\ University of Minnesota, Minneapolis, MN 55455, USA
}

\begin{abstract}
We consider the possibility that the soft supersymmetry-breaking parameters $m_{1 / 2}$ and $m_{0}$ of the MSSM are universal at some scale $M_{\text {in }}$ below the supersymmetric grand unification scale $M_{G U T}$, as might occur in scenarios where either the primordial supersymmetry-breaking mechanism or its communication to the observable sector involve a dynamical scale below $M_{G U T}$. We analyze the $\left(m_{1 / 2}, m_{0}\right)$ planes of such sub-GUT CMSSM models, noting the dependences of phenomenological, experimental and cosmological constraints on $M_{i n}$. In particular, we find that the coannihilation, focus-point and rapid-annihilation funnel regions of the GUT-scale CMSSM approach and merge when $M_{i n} \sim 10^{12} \mathrm{GeV}$. We discuss sparticle spectra and the possible sensitivity of LHC measurements to the value of $M_{i n}$.
\end{abstract}

CERN-PH-TH/2006-110

June 2006 


\section{Introduction}

The primary phenomenological reason for expecting supersymmetry to appear at the $\mathrm{TeV}$ scale is to ensure the naturalness of the hierarchy of mass scales in fundamental physics [1]. It is also known to facilitate the construction of simple Grand Unified Theories (GUTs) with no intermediate mass scale, if supersymmetry appears around the TeV scale [2]. These two motivations for low-energy supersymmetry arise specifically in theories with large GUT and Planck mass scales, and are supplemented by other motivations for low-energy supersymmetry, such as cold dark matter [3] and the existence of a light Higgs boson [4].

Supersymmetry is all very nice, but it must be broken, and there is no consensus how this occurs. Presumably the origin of supersymmetry breaking is with a gravitino mass in local supersymmetry [5], but the mechanism for gravitino mass generation is still unclear, as is the manner whereby this breaking is communicated to the supersymmetric partners of observable particles [6]. It is often supposed that supersymmetry is initially broken in some Polonyi or hidden sector of the theory $[7,8]$, and is then transmitted to the spartners of Standard Model particles by either gravitational-strength interactions or some high-scale gauge interactions.

In phenomenological treatments of supersymmetry, the effective observable magnitudes of these supersymmetry-breaking parameters at low scales are then calculated using the renormalization-group equations (RGEs) of the effective low-energy theory, which is typically taken to be the minimal supersymmetric extension of the Standard Model (MSSM) [9]. One often assumes that the soft supersymmetry-breaking parameters are universal at some high input scale, and we term the resulting constrained model the CMSSM [10-14]. However, it should be stressed that not all models of supersymmetry breaking, e.g., in string theory yield such universal input parameters [15].

There is also the question of what input scale should be used to initialize the renormalizationgroup running of the soft supersymmetry-breaking parameters. In most CMSSM studies, this is taken to be the supersymmetric GUT scale $M_{G U T} \sim 2 \times 10^{16} \mathrm{GeV}$, but this assumption may be questioned. In general, it should probably be taken as approximately equal to the lowest among the dynamical scales in the Polonyi or hidden sector where supersymmetry is originally broken, and the scales of the interactions that transmit this breaking to the observable MSSM particles.

One could well imagine scenarios in which the input scale is above the GUT scale, e.g., if supersymmetry breaking and its mediation are characterized by the Planck or the string scale. In this case, the soft supersymmetry-breaking gaugino masses $m_{1 / 2}$ would evolve to- 
gether down to the GUT scale, where they would still be universal, diverging at lower scales according to the conventional MSSM RGEs. On the other hand, the soft supersymmetrybreaking scalar masses $m_{0}$ would not in general be universal at the GUT scale $M_{G U T}$, but would be different for different GUT multiplets. For example, in conventional SU(5) the scalar masses of the spartners of the $d_{R}$ and $\ell_{L}$ would be identical, but different from those of the spartners of the $q_{L}, u_{R}$ and $e_{R}$, since they come from $\overline{5}$ and 10 representations, respectively. On the other hand, in flipped $\mathrm{SU}(5)$ the groupings would be $u_{R}, \ell_{L}$ and $q_{L}, d_{R}$, with the $e_{R}$ different again, whereas only in $\mathrm{SO}(10)$ would all the soft supersymmetry-breaking scalar masses of the quarks and leptons be universal (but not those of the Higgs bosons). These would be interesting scenarios to study, but are not the objects of this paper.

Here we study instead the equally (if not more) plausible case in which universality applies to the parameters $m_{1 / 2}$ and $m_{0}$ at some input scale below the GUT scale. This might occur if the scale at which supersymmetry is broken dynamically in some hidden sector is smaller than the $M_{G U T}$, for example due to the v.e.v. of some condensate that appears at a lower scale. A partial analogue may be the chiral-symmetry breaking quark condensate in QCD, which generates a 'soft' effective quark mass that 'dissolves' at scales above $\Lambda_{Q C D}$. Alternatively, perhaps 'hard' supersymmetry breaking in the hidden sector is communicated to the observable sector by loops of particles weighing less than $M_{G U T}$, which 'dissolve' at high scales. In any such sub-GUT CMSSM scenario, the gaugino masses would evolve in the same way as the gauge couplings at the leading (one-loop) level, but from a different starting point, so that their effective values at low energies would be less separated than they are in the usual GUT CMSSM scenario. Likewise, the effective values of the soft supersymmetrybreaking scalar masses at low energies would also be more similar in a sub-GUT CMSSM than in the usual scenario.

The renormalization of the gauge couplings would always be the same in sub-GUT CMSSM scenarios, and the successful coupling unification of supersymmetric GUTs would therefore be preserved. However, because the renormalizations of the soft supersymmetrybreaking parameters would differ in these scenarios, as we demonstrate and explain, the regions of the $\left(m_{1 / 2}, m_{0}\right)$ plane allowed by experiments and cosmology in such a sub-GUT CMSSM scenario may be very different from those allowed in the usual GUT CMSSM scenario. For example, the impact of the LEP constraint on the MSSM Higgs $h$ is more marked, because the reduced dependence on $m_{1 / 2}$ of $m_{\tilde{t}}$ (which largely controls $m_{h}$ ) implies that only values of $m_{1 / 2}$ larger than those required in the GUT CMSSM are allowed in a sub-GUT CMSSM.

However, the most dramatic aspect of a sub-GUT CMSSM scenario may be the altered 
form of the constraint imposed by the relic density of supersymmetric cold dark matter. We assume that $\mathrm{R}$ parity is conserved, so that the lightest supersymmetric particle (LSP) is stable, and hence should be present in the Universe today as a relic from the Big Bang. We further assume that the lightest supersymmetric particle (LSP) is the neutralino $\chi$. In the usual GUT CMSSM scenario, one may distinguish three well-separated, generic regions of the $\left(m_{1 / 2}, m_{0}\right)$ plane that are allowed by the dark matter constraint imposed by WMAP [16] on the relic $\chi$ density: the coannihilation region [17], the focus-point region [18] and the rapidannihilation funnel region $[11,19]$. In sub-GUT CMSSM models, these regions tend to merge in a striking way as the input supersymmetry-breaking scale is reduced. This behaviour is understandable, stemming from the relations between different MSSM particle masses. In the coannihilation region, the neutralino and lighter stau have very similar masses, whereas in the focus-point region $|\mu| \sim m_{W}$, and in the funnel region $m_{\chi} \sim m_{A} / 2$. Because of the different degrees of renormalization of the sparticle masses in sub-GUT CMSSM models, the relations between these masses and the underlying parameters $m_{1 / 2}$ and $m_{0}$ change, causing the three different regions to move and ultimately merge.

\section{Experimental, phenomenological and cosmological con- straints in the CMSSM}

We begin by briefly discussing the constraints imposed on a standard GUT CMSSM model. This will serve as a baseline for comparison with the sub-GUT CMSSM models which are the focus of this paper. In Fig. 1(a), we show the $\left(m_{1 / 2}, m_{0}\right)$ plane in the GUT CMSSM model for $\tan \beta=10$ and $m_{t}=172.5 \mathrm{GeV}$ [20]. Among the relevant phenomenological constraints shown are the limits on the chargino mass: $m_{\chi^{ \pm}}>104 \mathrm{GeV}[21]$, shown as the near-vertical (black) dashed line at low $m_{1 / 2}$, and on the Higgs mass: $m_{h}>114 \mathrm{GeV}$ [22], shown as the near-vertical (red) dot-dashed curve at $m_{1 / 2} \approx 400 \mathrm{GeV}^{1}$. Another phenomenological constraint is the requirement that the branching ratio for $b \rightarrow s \gamma$ be consistent with the experimental measurements [24]. These measurements agree with the Standard Model, and therefore provide bounds on MSSM particles [25] and hence the $\left(m_{1 / 2}, m_{0}\right)$ parameter space. At $\tan \beta=10$ and $\mu>0$, the bound due to $b \rightarrow s \gamma$ is weak, as is shown by the green shaded region at low $m_{1 / 2}$ and $m_{0}$. Typically, the $b \rightarrow s \gamma$ constraint is more important for $\mu<0$, but it is also relevant for $\mu>0$, particularly when $\tan \beta$ is large. Finally, we display with pink

\footnotetext{
${ }^{1}$ Here and throughout this paper, we use FeynHiggs [23] for the calculation of $m_{h}$. We do not allow for the possible theoretical and parametric errors in the FeynHiggs results, which would allow values of $m_{1 / 2} \sim 80 \mathrm{GeV}$ smaller for the value of $\tan \beta=10$ considered here.
} 
shading the regions of the $\left(m_{1 / 2}, m_{0}\right)$ plane that are favoured by the BNL measurement [26] of $g_{\mu}-2$ at the $2-\sigma$ level, as calculated in the Standard Model using $e^{+} e^{-}$data ${ }^{2}$.

As already mentioned, we assume that R parity is conserved, so that the LSP is stable, and we further assume that the LSP is the lightest neutralino $\chi$. Also shown as the turquoise shaded regions in Fig. 1 are the parts of the $\left(m_{1 / 2}, m_{0}\right)$ plane where the relic density of the neutralino LSP $\chi$ falls within the range preferred by WMAP, namely $0.085<\Omega_{C D M}<0.119$ at the $2-\sigma$ level [16]. The cosmological region shown in panel a) corresponds to the $\chi-\tilde{\tau}$ co-annihilation strip [17]. The 'bulk' region which existed formerly at small $m_{1 / 2}$ and $m_{0}$ is excluded for $\tan \beta=10$ with $m_{t}=172.5 \mathrm{GeV}$ by the Higgs mass bound.

There is an additional region of acceptable relic density in the GUT CMSSM model, known as the focus-point region [18], which is found at rather higher values of $m_{0}$. As $m_{0}$ is increased, the value of $\mu$ at the electroweak scale which is required in the GUT CMSSM to obey the electroweak symmetry breaking conditions eventually begins to drop. When $\mu \lesssim m_{1 / 2}$, the composition of the LSP gains a strong Higgsino component, and the relic density begins to drop precipitously. As $m_{0}$ is increased further, there is no longer any consistent solution for $\mu$. The focus-point region is not seen in panel a), since it occurs at $m_{0}>1000 \mathrm{GeV}$ for the value $m_{t}=172.5 \mathrm{GeV}$ assumed here. However, the focus-point region does appear in the sub-GUT CMSSM models discussed below.

Finally, another region of interest is that created by rapid annihilation via the directchannel pole mediated by the Higgs pseudoscalar $A$ when $m_{\chi} \sim \frac{1}{2} m_{A}[11,19]$. We recall that the heavier neutral scalar Higgs boson $H$ is almost degenerate with the pseudoscalar boson $A$, but plays a much less significant role in the annihilation process. Since the heavy scalar and pseudoscalar Higgs masses decrease as $\tan \beta$ increases, whilst $m_{\chi}$ is almost fixed by the value of $m_{1 / 2}$ and is largely independent of $m_{0}$, eventually $2 m_{\chi} \simeq m_{A}$ at any fixed value of $m_{1 / 2}$. The direct-channel annihilation then becomes rapid, yielding a 'funnel' of parameters with acceptable relic density, that extends to large $m_{1 / 2}$ and $m_{0}$ at large $\tan \beta$. This region is not present in the GUT CMSSM model at $\tan \beta=10$, but we will see that it appears when the input scale for supersymmetry breaking is reduced. The funnel due to rapid annihilation via the light Higgs scalar is excluded in this case by the chargino mass bound, as well as by the Higgs mass bound.

\footnotetext{
${ }^{2}$ The $\pm 1-\sigma$ range of the possible supersymmetric contribution to $g_{\mu}-2$ is indicated by dashed lines. In view of the uncertainty surrounding the Standard Model contribution to $g_{\mu}-2$, we consider the implementation of this constraint as purely indicative.
} 

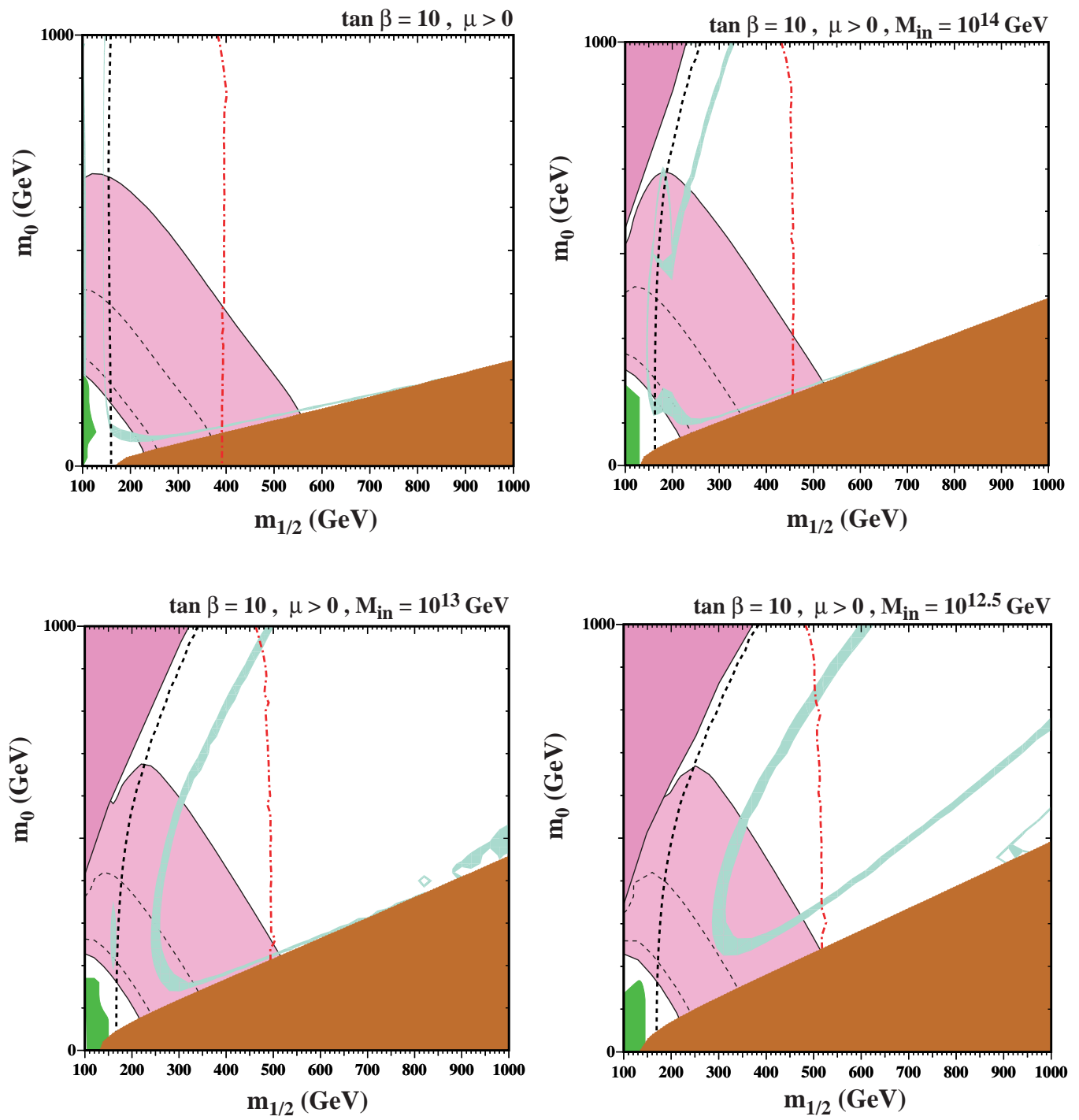

Figure 1: Examples of $\left(m_{1 / 2}, m_{0}\right)$ planes with $\tan \beta=10$ and $A_{0}=0$ but with different values of $M_{i n}$. (a) The CMSSM case with $M_{i n}=M_{G U T} \sim 2 \times 10^{16} \mathrm{GeV}$, (b) $M_{i n}=10^{14} \mathrm{GeV}$, (c) $M_{i n}=10^{13} \mathrm{GeV}$ and (d) $M_{i n}=10^{12.5} \mathrm{GeV}$. In each panel, we show the regions excluded by the LEP lower limits on MSSM particles, those ruled out by $b \rightarrow$ s $\gamma$ decay [24, 25] (medium green shading), and those excluded because the LSP would be charged (dark red shading). The region favoured by the WMAP range $\Omega_{C D M} h^{2}=0.1045_{-0.0095}^{+0.0072}$ has light turquoise shading. The region suggested by $g_{\mu}-2$ is medium (pink) shaded. 


\section{Lowering the universality scale for soft supersymme- try breaking}

We now explore the consequences of reducing below $M_{G U T}$ the scale at which universality is assumed for the supersymmetry-breaking parameters $m_{1 / 2}$ and $m_{0}$, as might occur if the underlying supersymmetry-breaking mechanism and/or the mechanism for communicating it to the observable sector are characterized by a dynamical scale $M_{i n}<M_{G U T}$. One could, in principle, imagine that the scales at which the $m_{1 / 2}$ and $m_{0}$ parameters are universal might be different, but we do not consider such a possibility here ${ }^{3}$.

As already mentioned, at the one-loop level the renormalizations of the gaugino masses $M_{a}(a=1,2,3)$ are identical with those of the corresponding gauge coupling strengths $\alpha_{a}$, so that in a sub-GUT CMSSM

$$
M_{a}(Q)=\frac{\alpha_{a}(Q)}{\alpha_{a}\left(M_{i n}\right)} M_{a}\left(M_{i n}\right),
$$

where the input gaugino masses $M_{a}\left(M_{i n}\right)=m_{1 / 2}$ by assumption. By comparison, in the usual GUT CMSSM, the values of the gaugino masses would already be different at the lower scale $M_{i n}: M_{a}\left(M_{i n}\right)=\left(\alpha_{a}\left(M_{i n}\right) / \alpha(G U T)\right) \times m_{1 / 2}$. Therefore, in the sub-GUT CMSSM scenario, the low-energy effective soft supersymmetry-breaking gaugino masses differ from each other by smaller amounts than in the usual GUT CMSSM.

The soft supersymmetry-breaking scalar masses of the different squark and slepton flavours and Higgs bosons $m_{0_{i}}$ are renormalized below the universality scale by both gauge interactions and Yukawa interactions. The latter are important for the stop squarks and the Higgs multiplet coupled to them, and for the sbottom squarks, stau sleptons and the other Higgs multiplet at large $\tan \beta$. The net effects of these renormalizations may be summarized as follows:

$$
m_{0_{i}}^{2}(Q)=m_{0}^{2}\left(M_{i n}\right)+C_{i}\left(Q, M_{i n}\right) m_{1 / 2}^{2},
$$

where the calculable renormalization coefficients $C_{i}\left(Q, M_{i n}\right) \rightarrow 0$ as $Q \rightarrow M_{i n}$, and, for $M_{\text {in }} \geq$ $10^{11} \mathrm{GeV}$ as explored here, $C_{i}\left(Q, M_{i n}\right) \rightarrow C_{i}\left(Q, M_{G U T}\right)$ monotonically as $M_{i n} \rightarrow M_{G U T}$. The coefficients $C_{i}\left(Q, M_{i n}\right)$ are positive for all the squarks and sleptons, but negative for the Higgs multiplet $H_{2}$ that is coupled to the top quark, and also for the other Higgs multiplet $H_{1}$ at large $\tan \beta$ when it has large couplings to the bottom quark and $\tau$ lepton. These negative corrections make possible dynamical electroweak symmetry breaking, if they drive the full quantity (2) for the corresponding Higgs multiplet negative at low energies. In our treatment

\footnotetext{
${ }^{3}$ We note, in passing, that we also assume universality at the same input scale for the soft trilinear supersymmetry-breaking parameters $A$, though this is not of great relevance for our discussion.
} 
of the sub-GUT CMSSM, we include these effects consistently in the electroweak vacuum conditions.

We see in Figs. 1] and 2 several features related to these renormalization effects. For example, as $M_{\text {in }}$ decreases, we see that the requirement that the LSP not be charged (shown as a brick-red shaded region), which imposes the bound $m_{\tilde{\tau}_{1}}>m_{\chi}$ (where $\tilde{\tau}_{1}$ is the lighter stau slepton), encroaches on the allowed region of the $\left(m_{1 / 2}, m_{0}\right)$ plane from the bottom-right corner. This can be understood from the RGE evolution. As $M_{i n}$ decreases, the ratio of the lightest neutralino mass to $m_{1 / 2}$ increases. Simultaneously, the coefficient $C_{\tilde{\tau}_{1}}$ decreases as $M_{\text {in }}$ decreases. Both effects go in the same direction of requiring a higher value of $m_{0}$ for a given value of $m_{1 / 2}$ in order to enforce $m_{\tilde{\tau}_{1}}>m_{\chi}$. We also see a (purple shaded) bound that encroaches on the allowed region of the $\left(m_{1 / 2}, m_{0}\right)$ plane from the top-left corner, which is due to the change in the electroweak vacuum conditions. The LEP chargino mass constraint lies just within this boundary, and further within the allowed region is a strip where $\Omega_{\chi}$ falls within the WMAP range ${ }^{4}$. This shift in this bound can also be traced directly to the diminished RGE evolution, and can be understood qualitatively from the tree-level solution for $\mu$ :

$$
\mu^{2}=\frac{\left(m_{1}^{2}-m_{2}^{2} \tan ^{2} \beta\right)}{\tan ^{2} \beta-1}-\frac{M_{Z}^{2}}{2}
$$

where $m_{1}$ and $m_{2}$ are the soft Higgs masses associated with $H_{1}$ and $H_{2}$ and the latter is coupled to the top sector ${ }^{5}$. For low and moderate values of $\tan \beta, m_{1}^{2}>0$ whilst $m_{2}^{2}<0$ at the weak scale. As $M_{i n}$ decreases, the running of $m_{1}$ and $m_{2}$ is suppressed and, as a result, the absolute values of both remain closer to $m_{0}$. Thus the value of $\mu$ at the weak scale is decreased for any fixed values of $m_{1 / 2}$ and $m_{0}$, and the line where $\mu^{2}$ changes sign is found at a lower value of $m_{0}$ for any fixed value of $m_{1 / 2}$. The purple shaded regions in Figs. 1 and 2 correspond to regions for which $\mu^{2}<0$, which are therefore unphysical.

Finally, we also see that the lower bound on $m_{1 / 2}$ due to the LEP Higgs constraint becomes more stringent as $M_{i n}$ decreases. This is because $m_{h}<m_{Z}$ at the tree level, with a renormalization that is dominated by a logarithmic dependence on $m_{\tilde{t}}$. In turn, we see from (2) that $m_{\tilde{t}}$ increases with $m_{1 / 2}$, at a rate that is suppressed as $M_{i n}$ is decreased. Thus, one requires a progressively higher value of $m_{1 / 2}$ in order to push the lightest CMSSM Higgs mass above the LEP lower limit $m_{h}>114 \mathrm{GeV}$.

\footnotetext{
${ }^{4}$ We return later to its detailed morphology and evolution with $M_{i n}$.

${ }^{5}$ Note that our results are based on full two-loop RGEs and not the simple explanatory approximations given in eqs. [1- 3
} 

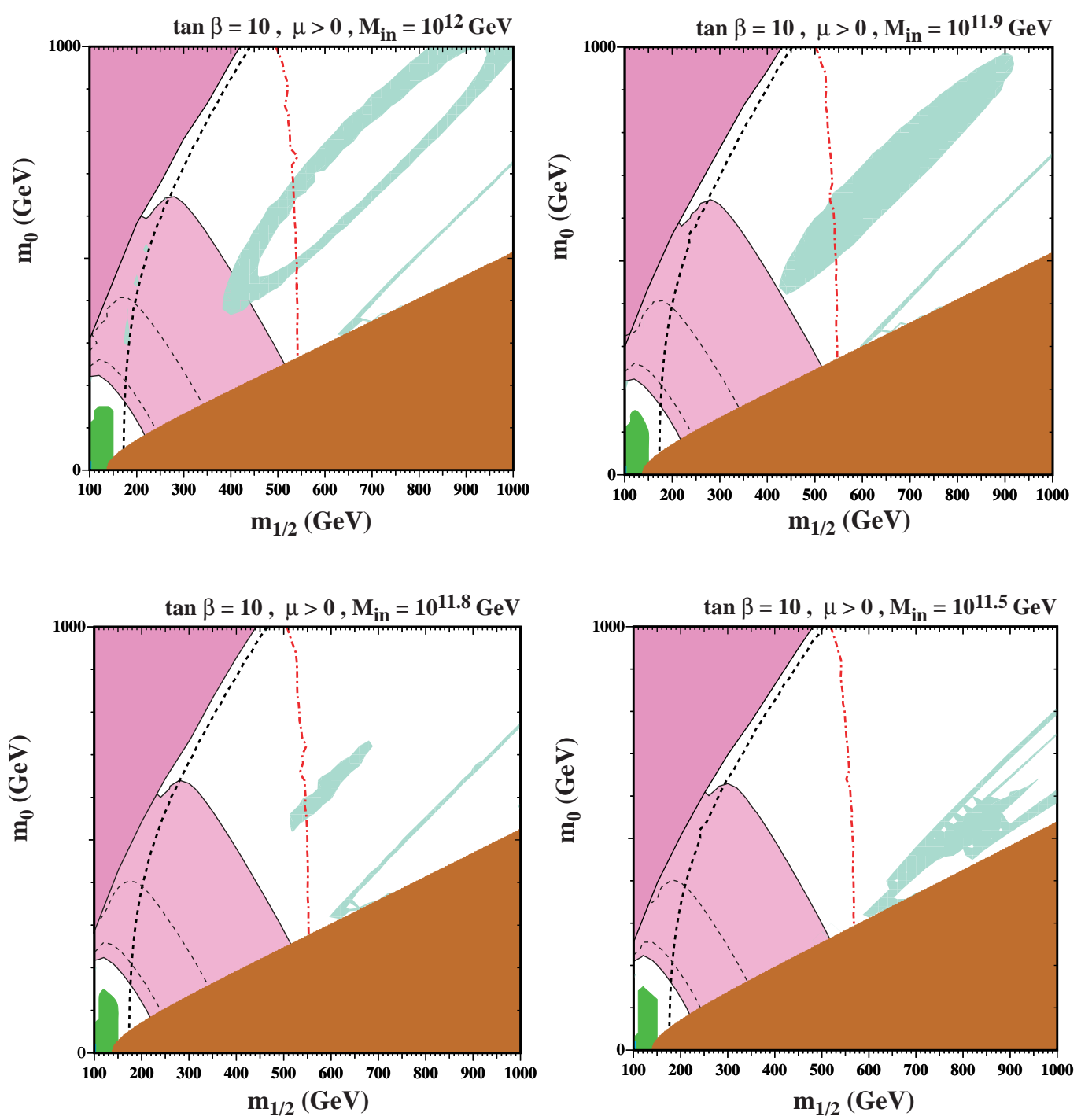

Figure 2: Examples of $\left(m_{1 / 2}, m_{0}\right)$ planes with $\tan \beta=10$ and $A_{0}=0$ but with different values of $M_{i n}$ (a) $M_{\text {in }}=10^{12} \mathrm{GeV}$, (b) $M_{i n}=10^{11.9} \mathrm{GeV}$, (c) $M_{i n}=10^{11.8} \mathrm{GeV}$ and (d) $M_{i n}=10^{11.5} \mathrm{GeV}$. In each panel, we show the regions excluded by the LEP lower limits on MSSM particles, those ruled out by $b \rightarrow$ s $\gamma$ decay [24,25] (medium green shading), and those excluded because the LSP would be charged (dark red shading). The region favoured by the WMAP range $\Omega_{C D M} h^{2}=0.1045_{-0.0095}^{+0.0072}$ has light turquoise shading. The region suggested by $g_{\mu}-2$ is medium (pink) shaded. 


\section{Evolution of the dark matter constraint}

We now discuss separately the evolving impact of the WMAP relic-density constraint as $M_{\text {in }}$ is decreased for fixed $\tan \beta=10$ and $\mu>0$. We see in the usual GUT CMSSM scenario in Fig. 11 the familiar feature of the $\chi-\tilde{\tau}_{1}$ coannihilation strip at low $m_{0}$, which extends from $m_{1 / 2} \sim 400 \mathrm{GeV}$ (where it is cut off by the $m_{h}$ constraint) up to $m_{1 / 2} \sim 900 \mathrm{GeV}$, where it drops down into the forbidden $\tilde{\tau}_{1}$ LSP region ${ }^{6}$. There is no funnel region for this value of $\tan \beta$, and the focus-point region is unseen at larger values of $m_{0}$. At low $m_{1 / 2} \sim 150 \mathrm{GeV}$, there is a strip where rapid annihilation via the $h$ pole would bring the $\chi$ density into the WMAP range which is, however, forbidden by the LEP chargino constraint and a fortiori the LEP Higgs constraint.

The picture starts changing already for $M_{i n}=10^{14} \mathrm{GeV}$, as seen in Fig. 1(b). The electroweak vacuum condition is visible at $\left(m_{1 / 2}, m_{0}\right) \sim(200,1000) \mathrm{GeV}$, with the chargino constraint close by, and a WMAP strip tracking its boundary with $m_{0} \sim 200 \mathrm{GeV}$ lower. This WMAP strip does not join directly with the coannihilation strip, but is instead deflected via a section of the rapid $h$ annihilation strip at $m_{1 / 2} \sim 150 \mathrm{GeV}$. This behaviour is linked to the $\chi \chi \rightarrow W W$ channel, which has a significant threshold in $m_{1 / 2}$, but whose importance varies with $m_{1 / 2}$ and $m_{0}$. The rate of variation of the relic density in this region is reflected in the thickness of the WMAP-allowed region. For example, if we follow the relic density at fixed $m_{0}=600 \mathrm{GeV}$, we find that at small $m_{1 / 2}$, the relic density is low due to the rapid annihilation through the light Higgs. As $m_{1 / 2}$ is increased, the density increases and at $m_{1 / 2} \simeq 170-190 \mathrm{GeV}$, the density is too high. However at slightly higher $m_{1 / 2}$, the $W W$ channel opens up, and because $\mu$ is lower relative to its value in the GUT-CMSSM, the relic density drops and becomes small at $m_{1 / 2} \lesssim 200 \mathrm{GeV}$. As one moves away from the forbidden triangle in the upper left, $\mu$ begins to increase, and the relic density again begins to increase so that the relic density is too large when $m_{1 / 2} \gtrsim 240 \mathrm{GeV}$. Thus, along this horizontal line, we have passed through three regions for which we match the WMAP relic density. The coannihilation strip is rather similar to that in the GUT CMSSM case shown in Fig. 1(a).

There is a more dramatic change for $M_{i n}=10^{13} \mathrm{GeV}$, as seen in Fig. 1(c). Not only has the electroweak vacuum constraint encroached further on the $\left(m_{1 / 2}, m_{0}\right)$ plane, but also the focus-point WMAP strip has receded further away from it, appearing at $m_{0} \sim 300 \mathrm{GeV}$ lower. Moreover, this focus-point strip now connects smoothly at $m_{1 / 2} \sim 250 \mathrm{GeV}$ with the $\chi-\tilde{\tau}_{1}$ coannihilation strip at low $m_{0}$. The coannihilation strip itself exhibits some broadening

\footnotetext{
${ }^{6}$ If the gravitino were light, the $\tilde{\tau}_{1}$ would become the NLSP in this region, and there would be an allowed region with gravitino dark matter, but we do not explore this possibility here.
} 
and embryonic bifurcation at $m_{1 / 2} \sim 1000 \mathrm{GeV}$, due to the approaching funnel.

The emerging picture is much clearer in Fig. 1(d), where $M_{i n}=10^{12.5} \mathrm{GeV}$. The focuspoint part of the WMAP strip has now separated further from the electroweak vacuum boundary, but also the linked 'coannihilation' portion of the WMAP strip has separated from the $\tilde{\tau}_{1}$ LSP boundary, by an amount that increases with $m_{1 / 2}$. In fact, we now recognize the region at large $m_{1 / 2}$ as the opening of a characteristic rapid $A, H$ annihilation funnel, of the type seen in the GUT CMSSM only when $\tan \beta \sim 50$ for $\mu>0$ as studied here. On the further side of the funnel, at $m_{1 / 2} \sim 900 \mathrm{GeV}$, we now see more clearly the bifurcation of the second funnel wall from the continuing coannihilation strip.

The changes described above accelerate as $M_{\text {in }}$ decreases further, as seen in Fig. 2. For $M_{i n}=10^{12} \mathrm{GeV}$, as seen in Fig. 2(a), the former focus-point, lower coannihilation and funnel regions merge into a WMAP ellipse that encloses just a small region where the $\chi$ relic density is too large. The further wall of the funnel and the continuation of the coannihilation strip form a well-developed 'vee' shape that extends to much larger values of $m_{1 / 2}$ than those shown here.

Even more strikingly, when $M_{i n}$ is reduced slightly to $10^{11.9} \mathrm{GeV}$, as shown in Fig. 2(b), the ellipse is now filled up. This is the culmination of a trend, noticeable already in Fig. 1, for the WMAP regions to broaden as well as merge as $M_{\text {in }}$ decreases. The possibility that the LSP relic density falls within the WMAP range therefore appears more 'natural'. Moreover, we see in Fig. 2(a), (b) that it is increasingly 'unlikely' that the relic density will exceed the WMAP range, whereas this appeared much more 'likely' in the GUT CMSSM case shown in Fig. 1(a). Whether one worries about the 'naturalness' of supersymmetric dark matter or not, it is nevertheless interesting that there is less cause for worry when $M_{\text {in }} \sim 10^{12} \mathrm{GeV}$.

The situation changes again with just a small change to $M_{i n}=10^{11.8} \mathrm{GeV}$, as seen in Fig. 1. The ellipse has now almost evaporated, with the relic density falling below the range favoured by WMAP over most of the visible part of the $\left(m_{1 / 2}, m_{0}\right)$ plane ${ }^{7}$. The only region with an excessive amount of cold dark matter is inside the 'vee' at large $m_{1 / 2}$. Note also, that the region favoured by the relic density no longer overlaps with the region preferred by the $g_{\mu}-2$ anomaly.

Finally, when $M_{i n}=10^{11.5} \mathrm{GeV}$, as shown in Fig. 1(d), the ellipse favoured by WMAP has disappeared completely We also notice that the large- $m_{1 / 2}$ 'vee' starts to fill in, with a new generic region of acceptable relic density now appearing. This is due, in particular, to the opening up of new annihilation channels such $(H, A)+Z, H^{ \pm}+W^{\mp}$ that are sufficient

\footnotetext{
${ }^{7}$ These regions would of course still be acceptable for cosmology, if there were another important source of cold dark matter.
} 
to bring the relic density down into the WMAP range. At lower values of $M_{i n} \rightarrow 10^{10} \mathrm{GeV}$ (not shown), the electroweak vacuum boundary continues to press downwards and the relic density is always below the favoured WMAP range for $m_{\chi}<m_{A} / 2$. The relic density lies within the WMAP range only along narrow strips close to the top and bottom of the 'vee' where $m_{\chi} \geq m_{A} / 2, m_{\tilde{\tau}_{1}}$. To better understand this behaviour, let us look at the density at fixed $m_{1 / 2}=900 \mathrm{GeV}$. At large $m_{0}$, the annihilation cross section is large dominated by the broad s-channel pole through the heavy Higgses, $H$ and $A$. As $m_{0}$ is lowered, $2 m_{\chi}$ becomes larger than $m_{A}$, and at $m_{0} \approx 700$, the WMAP density is attained. As one moves to lower $m_{0}$, away from the pole, the relic density increases, but the heavy Higgs masses decrease opening up the $H^{ \pm}+W^{\mp}$ channel when $m_{0} \approx 630 \mathrm{GeV}$ and the $(H, A)+Z$ at slightly lower $m_{0}$. In this region of the parameter space, the $s$-wave annihilation cross section is dominant and decreases as $m_{0}$ is lowered, so there is a modest increase in the density and the WMAP value is obtained again when $m_{0} \lesssim 600 \mathrm{GeV}$. At still lower $m_{0}$, yet another channel opens up. At $m_{0} \lesssim 560 \mathrm{GeV}$, the $h, A$ channel is open and the density once again drops below the WMAP value. As we continue to move off of the Higgs funnel, the $h, A$ contribution slowly decreases and the density rises and surpasses the WMAP value. At this value of $m_{1 / 2}$, we are past the endpoint of $\chi-\tilde{\tau}$ coannihilation and the density is too large as we enter the $\tilde{\tau}$ LSP region.

If we continue to lower the supersymmetry breaking input scale, $M_{i n}$, we find that the region seen in Fig. 11(d) begins to evaporate. At $M_{i n}=10^{11.2} \mathrm{GeV}$, it is gone, but the $\chi-\tilde{\tau}$ coannihilation region has returned for $M_{1 / 2} \gtrsim 600 \mathrm{GeV}$. The lower end of the coannihilation region continues to move to higher $M_{1 / 2}$ as $M_{i n}$ is decreased, so that when $M_{i n}<10^{10} \mathrm{GeV}$, the lower end of the coannihilation region is at $M_{1 / 2} \approx 900 \mathrm{GeV}$.

\section{$5 \quad$ Evolution of sparticle masses}

We now discuss the extent to which the results presented in the previous Section can be understood in terms of the evolution of sparticle masses with $M_{i n}$, and the corresponding implications for and of sparticle measurements at colliders such as the LHC.

We display in Fig. 3 two examples of the evolution of sparticle mass parameters with $M_{i n}$ in the focus-point region. Panel (a) is for $\left(m_{1 / 2}, m_{0}\right)=(200,1000) \mathrm{GeV}$, and panel (b) for $\left(m_{1 / 2}, m_{0}\right)=(500,1000) \mathrm{GeV}$. In each case, we show the evolution of the unmixed electroweak gaugino mass $M_{1}$ (blue dotted lines), the Higgs soft mass represented by $\operatorname{sgn}\left(m_{2}^{2}\right)\left(\sqrt{\left|m_{2}^{2}\right|}\right)$ (turquoise dot-dashed lines), the absolute value of $\mu$ (red dashed lines) and the LSP mass $m_{\chi}$ (solid black line). We see that, as $M_{i n}$ decreases from the GUT 
value of $2 \times 10^{16} \mathrm{GeV}$, both $\left|\sqrt{m_{2}^{2}}\right|$ and particularly $|\mu|$ plummet precipitously, whereas the gaugino masses $M_{1,2}$ evolve more slowly. In the GUT CMSSM, $m_{\chi}$ is essentially equal to $M_{1}$, but this changes as $M_{i n}$ decreases, and $m_{\chi}$ is given by $|\mu|$ when this is small. In both the examples shown, the first disaster to occur as $M_{i n}$ decreases is that $|\mu|$ vanishes, which marks the boundary of the region of the $\left(m_{1 / 2}, m_{0}\right)$ plane allowed by the electroweak vacuum conditions. The disallowed regions are shaded (purple): this boundary reaches the point $\left(m_{1 / 2}, m_{0}\right)=(200,1000) \mathrm{GeV}$ shown in panel (a) when $M_{i n} \sim 10^{14.5} \mathrm{GeV}$, whereas Armageddon is postponed until $M_{i n} \sim 10^{11.4} \mathrm{GeV}$ for the point $\left(m_{1 / 2}, m_{0}\right)=(500,1000) \mathrm{GeV}$ shown in panel (b). In both the cases studied, $\sqrt{\left|m_{2}^{2}\right|}$ does not vanish until well inside the region disallowed by the electroweak vacuum conditions. We have seen the consequences of this behavior in Figs. 1 and 2 as the encroachment of the region where the electroweak symmetry breaking conditions are not obeyed.

In panel (a), the relic neutralino density exceeds the WMAP upper limit in the GUT CMSSM, and the relic density falls as $M_{i n}$ decreases. There is a narrow range of $M_{i n} \sim$ $10^{15} \mathrm{GeV}$ where the density falls within the favoured WMAP range, and it then falls to zero as $|\mu|$ and hence $m_{\chi}$ vanishes. In panel (b), there is a similar sequence of events, with the WMAP range attained at a lower value of $M_{i n} \sim 10^{13.2} \mathrm{GeV}$.

Panels (c) and (d) of Fig. 3 provide analogous displays of the evolution of mass parameters with $M_{i n}$ in the funnel region, for $\left(m_{1 / 2}, m_{0}\right)=(1000,400) \mathrm{GeV}$ and $\left(m_{1 / 2}, m_{0}\right)=$ $(1000,800) \mathrm{GeV}$, respectively. Here, in addition to $M_{1}, \mu,\left|\sqrt{m_{2}^{2}}\right|$ and $m_{\chi}$, we also plot $m_{\tilde{\tau}_{1}}$ and $m_{A} / 2$. The evolution of $m_{\tilde{\tau}_{1}}$ is undramatic. As in panels (a) and (b), the physical region is bounded by the vanishing of $\mu$ and hence $m_{\chi}$, which occurs at $M_{i n} \sim 10^{6.5} \mathrm{GeV}$ and $M_{i n} \sim 10^{8.3} \mathrm{GeV}$ in cases (c) and (d), respectively. As in the cases (a) and (b), the LSP mass tracks $M_{1}$ at large $M_{i n}$ and then $\mu$ at smaller $M_{i n}$ after the values of $\mu$ and $M_{1}$ cross.

Among the more interesting aspects of panels (c) and (d) are the comparisons between $m_{\chi}$ and $m_{\tilde{\tau}_{1}}$, on the one hand, and between $m_{\chi}$ and $m_{A} / 2$, on the other hand. In panel (c), we see that $m_{\chi}$ rises above $m_{\tilde{\tau}_{1}}$ (which is unacceptable) when $M_{\text {in }}$ falls to $\sim 10^{14} \mathrm{GeV}$, a feature visible also in panel (b) of Fig. 1] where we notice that the point $\left(m_{1 / 2}, m_{0}\right)=(1000,400) \mathrm{GeV}$ sits on the boundary of the stau LSP region for this value of $M_{i n}$. We also note that $m_{\chi}$ falls (with $\mu$ ) below $m_{\tilde{\tau}_{1}}$ when $M_{i n}<10^{9} \mathrm{GeV}$, an effect not visible in our previous scans of the $\left(m_{1 / 2}, m_{0}\right)$ planes in Figs. 1 and 2, where we only considered $M_{i n} \geq 10^{11.5} \mathrm{GeV}$. In panel (d), we again see the crossover from $m_{\chi} \sim M_{1}$ to $m_{\chi} \sim \mu$, whereas $m_{\tilde{\tau}_{1}}>m_{\chi}$ in this case.

Comparing now $m_{\chi}$ with $m_{A} / 2$, we see in panel (c) that in the case $\left(m_{1 / 2}, m_{0}\right)=$ $(1000,400) \mathrm{GeV}$ they become equal only in the stau LSP region when $M_{i n} \sim 10^{13} \mathrm{GeV}$, whereas in the case $\left(m_{1 / 2}, m_{0}\right)=(1000,800) \mathrm{GeV}$ shown in panel (d) $m_{\chi}$ and $m_{A} / 2$ be- 

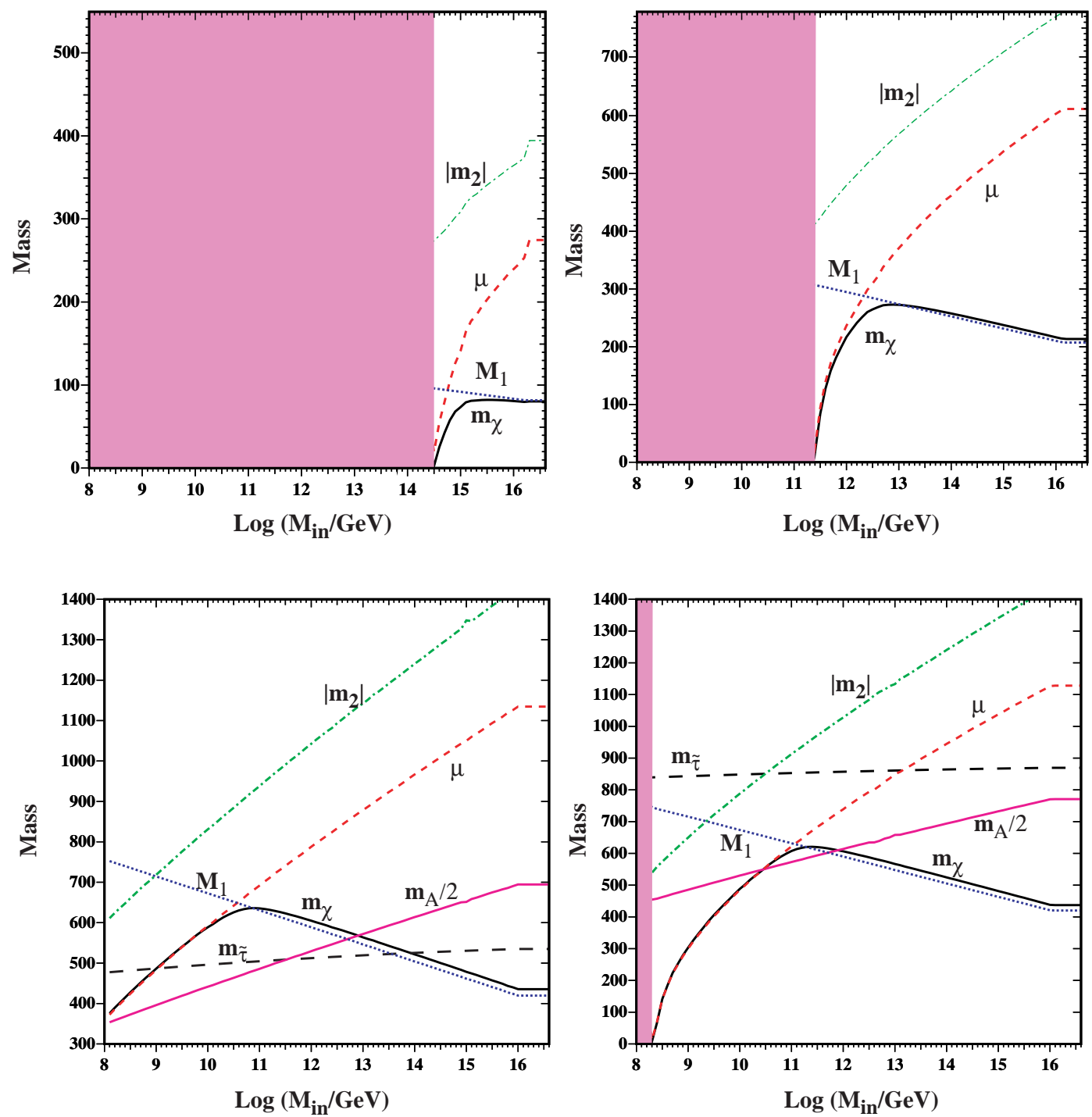

Figure 3: Mass parameters as functions of $M_{\text {in }}$ in the focus-point region, for $(a)\left(m_{1 / 2}, m_{0}\right)=$ $(200,1000) \mathrm{GeV}$, and $(b)\left(m_{1 / 2}, m_{0}\right)=(500,1000) \mathrm{GeV}$, and in the funnel region for (c) $\left(m_{1 / 2}, m_{0}\right)=(1000,400) \mathrm{GeV}$, and $(d)\left(m_{1 / 2}, m_{0}\right)=(1000,800) \mathrm{GeV}$. 
come equal twice, when $M_{i n} \sim 10^{12}$ and $10^{10.5} \mathrm{GeV}$, and $m_{\chi}$ and $m_{A} / 2$ are quite similar for intermediate and adjacent values of $M_{i n}$.

Since the relation between $m_{\chi}$ and $m_{\tilde{\tau}_{1}}$ is very important for coannihilation, and that between $m_{\chi}$ and $m_{A} / 2$ is very important for the rapid-annihilation funnel, these crossover patterns have important effects on the relic $\chi$ density, and enable us to understand some features of Figs. 11 and 2, Specifically, for $\left(m_{1 / 2}, m_{0}\right)=(1000,400) \mathrm{GeV}$ as shown in panel (c) of Fig. 3, the approach towards $m_{\chi}=m_{\tilde{\tau}_{1}}$ as $M_{i n} \rightarrow 10^{14} \mathrm{GeV}$ is responsible for a significant reduction in the dark matter density. The relic density is also reduced for the case $\left(m_{1 / 2}, m_{0}\right)=(1000,800) \mathrm{GeV}$ shown in panel (d) of Fig. 3] as $M_{\text {in }} \rightarrow 10^{12.5} \mathrm{GeV}$, as also seen in Fig. 1. The relic density then remains below the range favoured by WMAP as $M_{\text {in }} \rightarrow 10^{11.8} \mathrm{GeV}$, as seen in the first three panels of Fig. 2. On the other hand, the density rises to the favoured WMAP range when $M_{i n}=10^{11.5} \mathrm{GeV}$, and would even exceed the WMAP range for smaller values of $M_{i n}$. This is because $m_{\chi}$ is now greater than $m_{A} / 2$. However, we expect the density to fall again as $M_{i n}$ decreases further and $m_{\chi}$ decreases again and crosses $m_{A} / 2$ a second time.

\section{Implications for collider searches}

It is clear that the prospects for searches for supersymmetry at the LHC and other colliders depend on the value of $M_{i n}$ assumed. One may also ask to what extent collider measurements could be used to extract the value of $M_{i n}$, at least within a specific CMSSM framework. These are complicated issues whose full investigation would extend far beyond the scope of this exploratory study. Here we restrict our attention to two specific scans across the $\left(m_{1 / 2}, m_{0}\right)$ plane for $\tan \beta=10$ and $\mu>0$ as functions of $M_{i n}$, shown in Fig. 4. In scan (a), we first fix $m_{1 / 2}=700 \mathrm{GeV}$ and then, for each value of $M_{i n}$, find the values(s) of $m_{0}$ that yield a relic density within the range favoured by WMAP. Then, for each of these WMAPcompatible choices of $m_{0}$, we calculate the masses of some interesting sparticles, namely $\chi, \tilde{\tau}_{1}, \chi_{2}, \tilde{q}_{R}$ and $\tilde{g}$ and finally we plot their dependences on $M_{i n}$. In scan (b), we instead first fix $m_{0}=700 \mathrm{GeV}$, then find, for each value of $M_{i n}$, the value(s) of $m_{1 / 2}$ yielding the WMAP relic density, and finally plot the same set of masses as functions of $M_{i n}$.

In the case of the first scan at fixed $m_{1 / 2}=700 \mathrm{GeV}$ shown in Fig. U(a), as $M_{\text {in }}$ decreases from $2 \times 10^{16} \mathrm{GeV}$ towards $10^{13} \mathrm{GeV}$, we see that $m_{\tilde{g}}$ and $m_{\tilde{q}_{R}}$ decrease gradually, whereas $m_{\chi}, m_{\tilde{\tau}_{1}}$ and $m_{\chi_{2}}$ increase gradually. The behaviours of $m_{\tilde{g}}$ and $m_{\chi}$ are simply due to their reduced mass renormalizations as $M_{i n}$ decreases. In the case of $m_{\tilde{\tau}_{1}}$, at large $M_{i n}$, one must choose $m_{0}$ to lie within the WMAP coannihilation strip, so that the relic density remains 

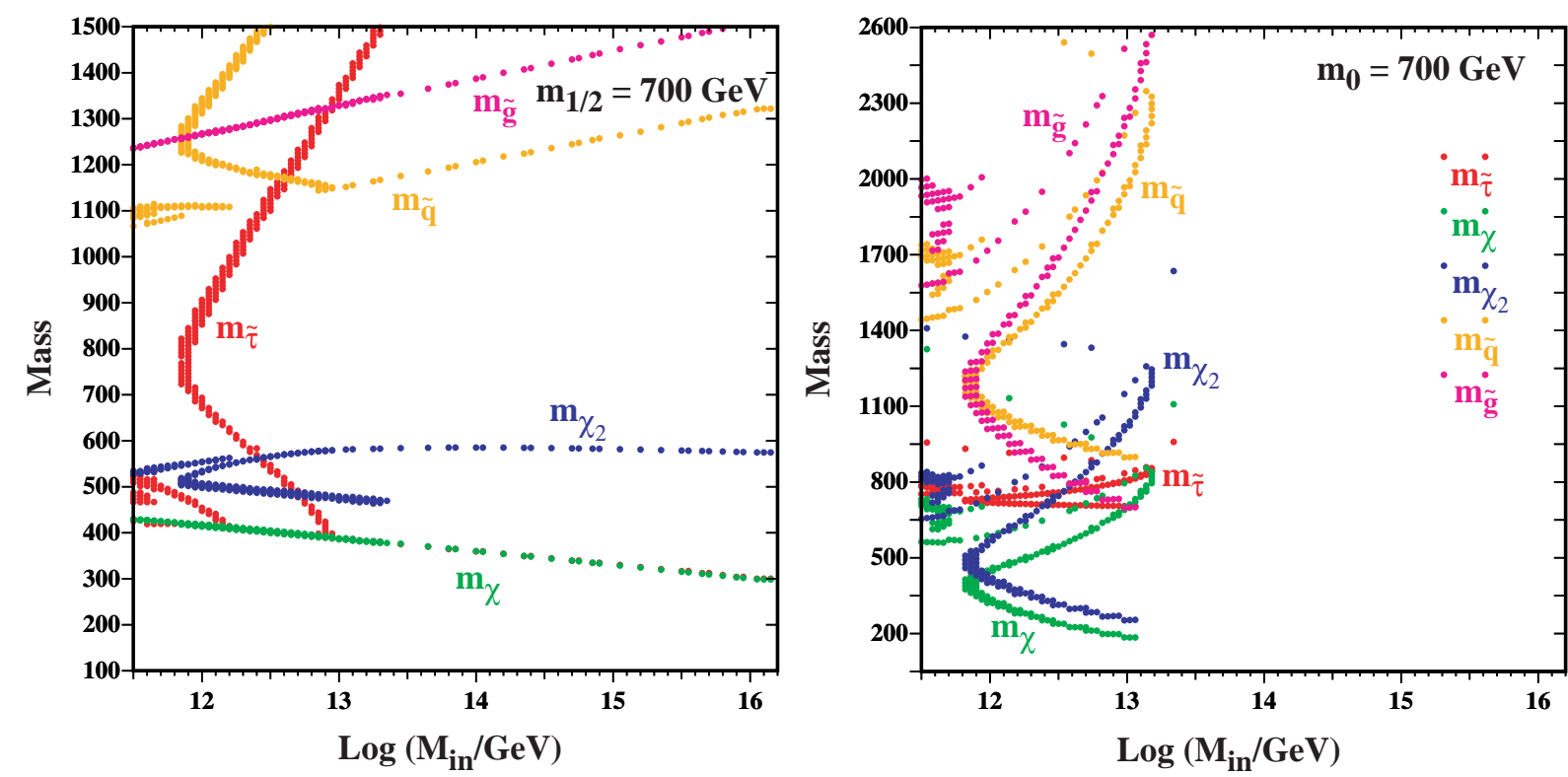

Figure 4: Sparticle masses for sub-GUT CMSSM models chosen to be compatible with the WMAP relic-density constraint for $\tan \beta=10, A=0, \mu>0$ and (a) $m_{1 / 2}=700 \mathrm{GeV}$, (b) $m_{0}=700 \mathrm{GeV}$. For each value of $M_{\text {in }}$, we choose (a) $m_{0}$ and (b) $m_{1 / 2}$ so as to respect $W M A P$, and then plot the corresponding sparticle masses as functions of $M_{i n}$.

within the allowed range. This requires $m_{\tilde{\tau}_{1}}$ to be only very slightly larger than $m_{\chi}{ }^{8}$, so it also increases as $M_{i n}$ decreases. In the case of $m_{\tilde{q}_{R}}$, there are effects due to both the reduced mass renormalization and the WMAP-induced change in $m_{0}$, the former being dominant. A new phenomenon appears as $M_{i n} \rightarrow 10^{13} \mathrm{GeV}$, namely, as seen in Fig. 1(d), the WMAP strip at small $m_{0}$ moves away from the coannihilation limit, and $m_{\tilde{\tau}_{1}}$ increases much more rapidly than $m_{\chi}$. Also, a new branch of the WMAP strip appears ${ }^{9}$ at large $m_{0}$, in which $m_{\tilde{\tau}_{1}}$ decreases as $M_{i n} \rightarrow 10^{12} \mathrm{GeV}$ : similar behaviour is apparent for $m_{\tilde{q}_{R}}$. For points in the upper $m_{0}$ branch, the $\chi_{2}$ has a lower mass and is predominantly Higgsino in content, whereas in the lower $m_{0}$ branch the $\chi_{2}$ is mostly wino. When $M_{i n} \sim 10^{12} \mathrm{GeV}$ as shown in Fig. 2, the two branches of the WMAP strip merge, as do the two possible values of $m_{\tilde{\tau}_{1}}, m_{\tilde{q}_{R}}$ and $m_{\chi_{2}}$. However, appearing already at $M_{i n}$ slightly larger than $10^{12} \mathrm{GeV}$, we see new, somewhat lower ranges of allowed values of $m_{\tilde{\tau}_{1}}$ and $m_{\tilde{q}_{R}}$ (and higher values of $\left.m_{\chi_{2}}\right)$, which correspond to the wedge of allowed $m_{0}$ values inside the 'vee' visible in Fig. 1(d) for $m_{1 / 2}$ beyond the rapid-annihilation funnel. It is apparent that the spectra allowed by

\footnotetext{
${ }^{8}$ For this reason, the (red) $\tilde{\tau}_{1}$ points are scarcely visible along the (green) $\chi$ line.

${ }^{9}$ This branch, associated with the focus point, exists at larger $M_{i n}$ as well, but it does not appear in our scan, because it only extends to $m_{0}=1500 \mathrm{GeV}$.
} 
WMAP are very sensitive to the assumed value of $M_{i n}$. For example, a determination of the ratio $m_{\chi} / m_{\tilde{g}}$ with an accuracy of $4 \%$ (which may be possible at the LHC) would by itself fix $M_{i n}$ to within an order of magnitude, in the restricted set of models considered here.

In the case of the second scan at $m_{0}=700 \mathrm{GeV}$, we see in Fig. 1 that due to the Higgs mass bound (we use here the value of $112 \mathrm{GeV}$ calculated using FeynHiggs, so as to account for theoretical uncertainties), a suitable WMAP strip appears only when $M_{i n} \lesssim 10^{13} \mathrm{GeV}$, and this is reflected in the disappearance of the sparticle mass lines just above $M_{\text {in }}=10^{13} \mathrm{GeV}$ in Fig. 4(b). As $M_{\text {in }}$ decreases, two of the branches for each sparticle mass merge. However, there are two other branches, one appearing near $M_{i n} \sim 10^{13} \mathrm{GeV}$ and the other closer to $M_{\text {in }} \sim 10^{12} \mathrm{GeV}$. These are due to the appearance of the WMAP-allowed 'vee' seen close to the $m_{\chi}=m_{\tilde{\tau}_{1}}$ line in Fig. 1(d) et seq.. In this case, we see that the WMAP-allowed values of the sparticle masses vary rapidly for $M_{i n} \in\left(10^{12}, 10^{13}\right) \mathrm{GeV}$. This another example how LHC measurements of sparticle masses would help fix the magnitude of $M_{i n}$ in this restricted set of models.

\section{Discussion}

We have presented a first exploration of the dependence of the $\left(m_{1 / 2}, m_{0}\right)$ plane for $\tan \beta=$ $10, A=0, \mu>0$ on the scale $M_{\text {in }}$ at which the input soft supersymmetry-breaking CMSSM mass parameters $m_{1 / 2}$ and $m_{0}$ are assumed to be universal. We have displayed and explained how the phenomenological, experimental and cosmological constraints vary with $M_{i n}$. In particular, we have shown that the morphology of the region favoured by the WMAP range of the relic density changes with $M_{i n}$. Specifically, the focus point region at large $m_{0}$ the coannihilation strip and the rapid-annihilation funnel at large $m_{1 / 2}$ approach each other and merge as $M_{\text {in }}$ decreases to $\sim 10^{12} \mathrm{GeV}$. Consequently, the values of the sparticle masses that would be compatible with WMAP depend on $M_{i n}$, and measurements at the LHC may be able to offer some hints about the value of $M_{\text {in }}$ within such sub-GUT CMSSM scenarios.

It is desirable to extend this discussion to other values of the CMSSM parameters $\tan \beta$ and $A$. It would also be interesting to extend this analysis to less constrained versions of the MSSM, such as models with non-universal Higgs masses, and also more constrained versions of the MSSM motivated by minimal supergravity. It would also be valuable to extend the brief discussion given here of the corresponding spectra and the prospects for the LHC and ILC to 'measure' indirectly the value of $M_{i n}$. We plan to return to these issues in a future paper. 


\section{Acknowledgments}

The work of K.A.O. and P.S. was supported in part by DOE grant DE-FG02-94ER-40823.

\section{References}

[1] E. Witten, Nucl. Phys. B 188 (1981) 513; N. Sakai, Z. Phys. C 11 (1981) 153; S. Dimopoulos and H. Georgi, Nucl. Phys. B 193 (1981) 150; R. K. Kaul and P. Majumdar, Nucl. Phys. B 199 (1982) 36.

[2] J. R. Ellis, S. Kelley and D. V. Nanopoulos, Phys. Lett. B 260 (1991) 131; U. Amaldi, W. de Boer and H. Furstenau, Phys. Lett. B 260 (1991) 447; P. Langacker and M. x. Luo, Phys. Rev. D 44 (1991) 817; C. Giunti, C. W. Kim and U. W. Lee, Mod. Phys. Lett. A 6 (1991) 1745.

[3] J. Ellis, J.S. Hagelin, D.V. Nanopoulos, K.A. Olive and M. Srednicki, Nucl. Phys. B 238 (1984) 453; see also H. Goldberg, Phys. Rev. Lett. 50 (1983) 1419.

[4] J. R. Ellis, G. Ridolfi and F. Zwirner, Phys. Lett. B 257 (1991) 83; Phys. Lett. B 262 (1991) 477; A. Yamada, Phys. Lett. B 263, 233 (1991); M. Drees and M. M. Nojiri, Phys. Rev. D 45 (1992) 2482; P. H. Chankowski, S. Pokorski and J. Rosiek, Phys. Lett. B 274 (1992) 191; Phys. Lett. B 286 (1992) 307; A. Dabelstein, Z. Phys. C 67 (1995) 495 arXiv:hep-ph/9409375; M. Carena, J. R. Ellis, A. Pilaftsis and C. E. M. Wagner, Nucl. Phys. B 586 (2000) 92 arXiv:hep-ph/0003180; A. Katsikatsou, A. B. Lahanas, D. V. Nanopoulos and V. C. Spanos, Phys. Lett. B 501 (2001) 69 arXiv:hep-ph/0011370.

[5] E. Cremmer, B. Julia, J. Scherk, S. Ferrara, L. Girardello and P. Van Nieuwenhuizen, Phys. Lett. 79B (1978) 231; and Nucl. Phys. B147 (1979) 105; E. Cremmer, S. Ferrara, L. Girardello and A. Van Proeyen, Phys. Lett. 116B (1982) 231; and Nucl. Phys. B212 (1983) 413; R. Arnowitt, A.H. Chamseddine and P. Nath, Phys. Rev. Lett. 49 (1982) 970; 50 (1983) 232 and Phys. Lett. 121B (1983) 33; J. Bagger and E. Witten, Phys. Lett. 115B (1982) 202 and 118B (1982) 103; J. Bagger, Nucl. Phys. B211 (1983) 302.

[6] For reviews, see: H. P. Nilles, Phys. Rep. 110 (1984) 1; A. Brignole, L. E. Ibanez and C. Munoz, arXiv:hep-ph/9707209, published in Perspectives on supersymmetry, ed. G. L. Kane, pp. 125-148. 
[7] J. Polonyi, Budapest preprint KFKI-1977-93 (1977).

[8] R. Barbieri, S. Ferrara and C.A. Savoy, Phys. Lett. 119B (1982) 343.

[9] For a review see e.g. H. E. Haber and G. L. Kane, Phys. Rept. 117 (1985) 75.

[10] M. Drees and M. M. Nojiri, Phys. Rev. D 47 (1993) 376 arXiv:hep-ph/9207234; H. Baer and M. Brhlik, Phys. Rev. D 53 (1996) 597 arXiv:hep-ph/9508321 J. R. Ellis, T. Falk, K. A. Olive and M. Schmitt, Phys. Lett. B 388 (1996) 97 arXiv:hep-ph/9607292; Phys. Lett. B 413 (1997) 355 arXiv:hep-ph/9705444; J. R. Ellis, T. Falk, G. Ganis, K. A. Olive and M. Schmitt, Phys. Rev. D 58 (1998) 095002 arXiv:hep-ph/9801445; J. R. Ellis, T. Falk, G. Ganis and K. A. Olive, Phys. Rev. D 62 (2000) 075010 arXiv:hep-ph/0004169; V. D. Barger and C. Kao, Phys. Rev. D 57 (1998) 3131 arXiv:hep-ph/9704403.

[11] J. R. Ellis, T. Falk, G. Ganis, K. A. Olive and M. Srednicki, Phys. Lett. B 510 (2001) 236 arXiv:hep-ph/0102098.

[12] V. D. Barger and C. Kao, Phys. Lett. B 518 (2001) 117 arXiv:hep-ph/0106189; L. Roszkowski, R. Ruiz de Austri and T. Nihei, JHEP 0108 (2001) 024 arXiv:hep-ph/0106334; A. B. Lahanas and V. C. Spanos, Eur. Phys. J. C 23 (2002) 185 arXiv:hep-ph/0106345; A. Djouadi, M. Drees and J. L. Kneur, JHEP 0108 (2001) 055 arXiv:hep-ph/0107316; U. Chattopadhyay, A. Corsetti and P. Nath, Phys. Rev. D 66 (2002) 035003 arXiv:hep-ph/0201001; J. R. Ellis, K. A. Olive and Y. Santoso, New Jour. Phys. 4 (2002) 32 arXiv:hep-ph/0202110; H. Baer, C. Balazs, A. Belyaev, J. K. Mizukoshi, X. Tata and Y. Wang, JHEP 0207 (2002) 050 arXiv:hep-ph/0205325; R. Arnowitt and B. Dutta, arXiv:hep-ph/0211417.

[13] J. R. Ellis, K. A. Olive, Y. Santoso and V. C. Spanos, Phys. Lett. B 565 (2003) 176 arXiv:hep-ph/0303043; H. Baer and C. Balazs, arXiv:hep-ph/0303114; A. B. Lahanas and D. V. Nanopoulos, arXiv:hep-ph/0303130; U. Chattopadhyay, A. Corsetti and P. Nath, arXiv:hep-ph/0303201; C. Munoz, hep-ph/0309346.

[14] J. R. Ellis, K. A. Olive, Y. Santoso and V. C. Spanos, Phys. Rev. D 69 (2004) 095004 arXiv:hep-ph/0310356; J. Ellis, S. Heinemeyer, K. Olive and G. Weiglein, JHEP 0502 013, hep-ph/0411216; B. Allanach and C. Lester, Phys. Rev. D 73 (2006) 015013, hep-ph/0507283 B. Allanach, hep-ph/0601089 R. de Austri, R. Trotta and L. Roszkowski, hep-ph/0602028; J. Ellis, S. Heinemeyer, K. A. Olive and G. Weiglein, arXiv:hep-ph/0602220. 
[15] A. Corsetti and P. Nath, Phys. Rev. D 64 (2001) 125010 arXiv:hep-ph/0003186; R. Arnowitt, B. Dutta and Y. Santoso, Nucl. Phys. B $606 \quad$ (2001) 59 arXiv:hep-ph/0102181; D. G. Cerdeno, E. Gabrielli, S. Khalil, C. Munoz, E. TorrenteLujan and E. Torrente-Lujan, Nucl. Phys. B 603 (2001) 231 arXiv:hep-ph/0102270.

[16] D. N. Spergel et al., arXiv:astro-ph/0603449.

[17] J. R. Ellis, T. Falk and K. A. Olive, Phys. Lett. B 444 (1998) 367 arXiv:hep-ph/9810360; J. R. Ellis, T. Falk, K. A. Olive and M. Srednicki, Astropart. Phys. 13 (2000) 181 [Erratum-ibid. 15 (2001) 413] arXiv:hep-ph/9905481; R. Arnowitt, B. Dutta and Y. Santoso, Nucl. Phys. B 606 (2001) 59 arXiv:hep-ph/0102181; M. E. Gómez, G. Lazarides and C. Pallis, Phys. Rev. D D61 (2000) 123512 arXiv:hep-ph/9907261; Phys. Lett. B487 (2000) 313 arXiv:hep-ph/0004028; Nucl. Phys. B B638 (2002) 165 arXiv:hep-ph/0203131; T. Nihei, L. Roszkowski and R. Ruiz de Austri, JHEP 0207 (2002) 024 arXiv:hep-ph/0206266.

[18] J. L. Feng, K. T. Matchev and T. Moroi, Phys. Rev. Lett. 84 (2000) 2322; J. L. Feng, K. T. Matchev and T. Moroi, Phys. Rev. D61 (2000) 075005; J. L. Feng, K. T. Matchev and F. Wilczek, Phys. Lett. B482 (2000) 388.

[19] H. Baer and M. Brhlik, Phys. Rev. D 53 (1996) 597 arXiv:hep-ph/9508321; H. Baer, M. Brhlik, M. A. Diaz, J. Ferrandis, P. Mercadante, P. Quintana and X. Tata, Phys. Rev. D 63 (2001) 015007 arXiv:hep-ph/0005027; A. B. Lahanas and V. C. Spanos, Eur. Phys. J. C 23 (2002) 185 arXiv:hep-ph/0106345.

[20] Tevatron Electroweak Working Group, Combination of CDF and D0 results on the mass of the top quark, arXiv:hep-ex/0603039

[21] Joint LEP 2 Supersymmetry Working Group, Combined LEP Chargino Results up to $208 \mathrm{GeV}$, http://lepsusy.web.cern.ch/lepsusy/www/inos_moriond01/charginos_pub.html.

[22] LEP Higgs Working Group for Higgs boson searches, OPAL Collaboration, ALEPH Collaboration, DELPHI Collaboration and L3 Collaboration, Phys. Lett. B 565 (2003) 61 arXiv:hep-ex/0306033. Search for neutral Higgs bosons at LEP, paper submitted to ICHEP04, Beijing, LHWG-NOTE-2004-01, ALEPH-2004-008, DELPHI-2004-042, L3NOTE-2820, OPAL-TN-744, http://lephiggs.web.cern.ch/LEPHIGGS/papers/August2004_MSSM/index.html 
[23] S. Heinemeyer, W. Hollik and G. Weiglein, Comput. Phys. Commun. 124 (2000) 76 arXiv:hep-ph/9812320; S. Heinemeyer, W. Hollik and G. Weiglein, Eur. Phys. J. C 9 (1999) 343 arXiv:hep-ph/9812472.

[24] S. Chen et al. [CLEO Collaboration], Phys. Rev. Lett. 87 (2001) 251807 arXiv:hep-ex/0108032; P. Koppenburg et al. [Belle Collaboration], Phys. Rev. Lett. 93 (2004) 061803 arXiv:hep-ex/0403004. B. Aubert et al. [BaBar Collaboration], arXiv:hep-ex/0207076.

[25] M. Ciuchini, G. Degrassi, P. Gambino and G. F. Giudice, Nucl. Phys. B 527 (1998) 21 arXiv:hep-ph/9710335; Nucl. Phys. B 534 (1998) 3 arXiv:hep-ph/9806308; C. Degrassi, P. Gambino and G. F. Giudice, JHEP 0012 (2000) 009 arXiv:hep-ph/0009337; M. Carena, D. Garcia, U. Nierste and C. E. Wagner, Phys. Lett. B 499 (2001) 141 arXiv:hep-ph/0010003; P. Gambino and M. Misiak, Nucl. Phys. B 611 (2001) 338; D. A. Demir and K. A. Olive, Phys. Rev. D 65 (2002) 034007 arXiv:hep-ph/0107329; F. Borzumati, C. Greub and Y. Yamada, Phys. Rev. D 69 (2004) 055005 arXiv:hep-ph/0311151; T. Hurth, Rev. Mod. Phys. 75 (2003) 1159 arXiv:hep-ph/0212304.

[26] G. W. Bennett et al. [Muon g-2 Collaboration], Phys. Rev. Lett. 92 (2004) 161802 arXiv:hep-ex/0401008; M. Davier, S. Eidelman, A. Hocker and Z. Zhang, Eur. Phys. J. C 31 (2003) 503 arXiv:hep-ph/0308213; K. Hagiwara, A. D. Martin, D. Nomura and T. Teubner, arXiv:hep-ph/0312250 J. F. de Trocóniz and F. J. Ynduráin, arXiv:hep-ph/0402285 K. Melnikov and A. Vainshtein, arXiv:hep-ph/0312226 M. Passera, arXiv:hep-ph/0411168. 